\title{
Raman Photoassociation of Bose-Fermi Mixtures and the Subsequent Prospects for Atom-Molecule Cooper Pairing
}

\author{
Matt Mackie, ${ }^{1,2}$ Olavi Dannenberg, ${ }^{2}$ Jyrki Piilo,, 1, , 田 Kalle-Antti Suominen, ${ }^{1,2}$ and Juha Javanainen ${ }^{3}$ \\ ${ }^{1}$ Department of Physics, University of Turku, FIN-20014 Turun yliopisto, Finland \\ ${ }^{2}$ Helsinki Institute of Physics, PL 64, FIN-00014 Helsingin yliopisto, Finland \\ ${ }^{3}$ Department of Physics, University of Connecticut, Storrs, Connecticut, 06269-3046
}

(Dated: November 21, 2018)

\begin{abstract}
We theoretically investigate Raman photoassociation of a degenerate Bose-Fermi mixture of atoms and the subsequent prospect for anomalous (Cooper) pairing between atoms and molecules. Stable fermionic molecules are created via free-bound-bound stimulated Raman adiabatic passage which, in contrast to purely bosonic systems, can occur in spite of collisions. With the leftover atomic condensate to enhance intrafermion interactions, the superfluid transition to atom-molecule Cooper pairs occurs at a temperature that is roughly an order of magnitude below what is currently feasible.
\end{abstract}

PACS numbers: 03.75.Ss, 05.30.Fk, 74.20.Mn, 21.10.-k

Photoassociation occurs when two atoms absorb a laser photon [1], thereby jumping from the free twoatom continuum to a bound molecular state. Neutralatom statistics is determined by the number of neutrons in the nucleus-odd for fermions and even for bosons. Similarly, the sum of the total number of neutrons in the nuclei of the constituent atoms determines neutralmolecule statistics. Molecules formed by photoassociation of two fermions will accordingly result in a boson, whereas fermionic molecules are born of a boson and a fermion. Given degenerate Bose-Fermi atoms, two questions arise: Will the atoms photoassociate with into an arbitrary number of stable Fermi molecules? If so, is it possible to realize atom-molecule Cooper pairing?

First introduced to explain superconductivity, anomalous quantum correlations between two degenerate electrons with equal and opposite momenta-Cooper pairsare due physically to an electron-electron attraction mediated by the exchange of lattice-vibration-generated phonons 2], and are a manifestation of fermionic superfluidity [3]. Anomalous pairing between different chemical species was immediately suggested to explain the larger excitation energy for nuclei with even rather than odd numbers of nucleons [4], although it turned out that interspecies pairing plays the dominant role. Today quantum matter optics offers a means to explore condensed-matter and nuclear physics by proxy, such as the pairing of fermions in atomic traps and nuclei [5].

Here we investigate Raman photoassociation [ 6, 7, $, 8,[9]$ of Bose-Fermi mixtures of atoms [10], and the subsequent prospects for Cooper pairing between different chemical species (i.e., atoms and molecules). First, we demonstrate that an arbitrary number of stable Fermi molecules can be created via fractional 11] stimulated Raman adiabatic passage (STIRAP [12]), which is feasible because, contrary to bosonic systems [8], collisional interactions

*Presently at School of Pure and Applied Physics, University of KwaZulu-Natal, Durban 4041, South Africa. can be negligible. Density fluctuations in the condensate leftover from the photoassociation process then replace the vibrating ion lattice of the superconductor [13], and the subsequent phonon exchange can enhance the intrafermion attraction. We find that a typical attraction is enhanced, but this enhancement is insufficient for a transition to atom-molecule Cooper pairs within reach of present ultracold technology.

We model a Bose-Fermi mixture of atoms coupled by heteronuclear photoassociation to electronically-excited Fermi molecules, which is favored over homonuclear transitions for well resolved resonances [14]. The excited molecules are themselves coupled by a second laser to electronically stable molecules. For a degenerate system, the bosonic [fermionic] atoms of mass $m_{0}\left[m_{+}\right]$are represented by the field $\psi_{0}(\mathbf{r}, t)\left[\psi_{+}(\mathbf{r}, t)\right]$, and the excited [stable] fermionic molecules of mass $m_{e}=m_{0}+m_{+}$ $\left[m_{-}=m_{e}\right]$ by the field $\psi_{e}(\mathbf{r}, t)\left[\psi_{-}(\mathbf{r}, t)\right]$, with the boson (fermion) field operator obeying commutation (anticommutation) relations. The Hamiltonian density for said non-ideal system is $\mathcal{H}=\mathcal{H}_{0}+\mathcal{H}_{I}$, where

$$
\begin{aligned}
\frac{\mathcal{H}_{0}}{\hbar}= & -\Delta \psi_{-}^{\dagger} \psi_{-}+(\delta-\Delta) \psi_{e}^{\dagger} \psi_{e}+\lambda_{+-} \psi_{+}^{\dagger} \psi_{-}^{\dagger} \psi_{-} \psi_{+} \\
& +\sum_{\sigma} \psi_{\sigma}^{\dagger}\left[-\frac{\hbar \nabla^{2}}{m_{\sigma}}-\mu_{\sigma}+\lambda_{0 \sigma} \psi_{0}^{\dagger} \psi_{0}\right] \psi_{\sigma}, \\
\frac{\mathcal{H}_{I}}{\hbar}= & -\frac{1}{2}\left[\left(\mathcal{K}_{+} \psi_{e}^{\dagger} \psi_{+} \psi_{0}+\Omega_{-} \psi_{e} \psi_{-}^{\dagger}\right)+\text { H.c. }\right] .
\end{aligned}
$$

The light-matter coupling due to laser $1(2)$ is $\mathcal{K}_{+}$ $\left(\Omega_{-}\right)$, and the intermediate (two-photon) laser detuning, basically the binding energy of the excited (stable) molecular state relative to the photodissociation threshold, is $\delta(\Delta)$. Particle trapping is implicit to the chemical potential $\hbar \mu_{\sigma}(\sigma=0, e, \pm)$, and explicit traps can be neglected for most practical purposes. Low-energy ( $s$-wave) collisions are accounted for by the boson-boson (boson-fermion, fermion-fermion) interaction strength $\lambda_{00}=2 \pi \hbar a_{00} / m_{0}\left(\lambda_{0 \pm}=4 \pi \hbar a_{0 \pm} / m_{0 \pm}^{*}\right.$, $\left.\lambda_{+-}=4 \pi \hbar a_{+-} / m_{+-}^{*}\right)$, with $a_{\sigma_{1} \sigma_{2}}$ the $s$-wave scattering 
length and $m_{\sigma_{1} \sigma_{2}}^{*}$ the reduced mass. Spontaneous decay, included as $\Im[\delta]=-\Gamma$, is generally large enough to justify the exclusion of excited-molecule collisions.

Consider now the process of stimulated Raman adiabatic passage from atoms to stable molecules. The key to STIRAP is counterintuitive pulse timing, meaning that the two lasers are adjusted so that in the beginning, when the population is in the initial state (atoms), the strongest coupling is between the intermediate and final states (excited and stable molecules), while in the end, when effectively everything is in the target state, the coupling between the initial and excited states is strongest. As the system passes from atoms to stable molecules, the state with the larger population is always weakly coupled to the electronically-excited molecular state, and the subsequently low (ideally zero) population reduces (eliminates) radiative losses.

An overview of our STIRAP model is now presented (see also Appendix). When the number of bosons is much greater than the number of fermions, $N_{B} \gg N_{F}$, the frequency scale for atom-molecule conversion is set by $\Omega_{+}=\sqrt{\rho_{B}} \mathcal{K}_{+}$. Although a maximum $N_{B}=100$ is used, qualitative scaling to large boson number is cinched by assuming a density, $\rho_{B}=5 \times 10^{14} \mathrm{~cm}^{-3}$, consistent with $N_{B}=1.3 \times 10^{6}$ Bose-condensed ${ }^{87} \mathrm{Rb}$ atoms in a trap with radial and axial frequencies $\omega_{r}=100 \times 2 \pi \mathrm{Hz}$ and $\omega_{a}=10 \times 2 \pi \mathrm{Hz}$. For this density, a ballpark peak value for the atom-molecule coupling is $\Omega_{+} \sim \Omega_{0}=2 \pi \mathrm{MHz}$. A typical spontaneous decay rate is $\Gamma=10 \times 2 \pi \mathrm{MHz}$. The (assumedly negative) Fermi atom-molecule scattering length is estimated as $\left|a_{+-}\right|=a_{00}=5.29 \mathrm{~nm}$. The number of fermions is restricted to $N_{F}=4$ for numerical ease, and large-particle-number scaling is again ensured by picking a density, $\rho_{F}=1.1 \times 10^{12} \mathrm{~cm}^{-3}$, consistent with $N_{F}=5 \times 10^{3}{ }^{40} \mathrm{~K}$ atoms in the same (mass-adjusted) trap as the bosons, so that $\Lambda_{+-}=\lambda_{+-} / V=5.81 \times 2 \pi \mathrm{Hz}$. Numerics are further eased by restricting collisions between fermions to a bare minimum: $\mathbf{k}_{1}+\mathbf{k}_{2}=\mathbf{k}_{3}+\mathbf{k}_{4}$; $\mathbf{k}_{1}=\mathbf{k}_{3}, \mathbf{k}_{2}=\mathbf{k}_{4}$. Also, $N_{B} \gg N_{F}$ means that collisions with condensate atoms can be neglected.

Of course, complete conversion would leave no atoms to form Cooper pairs with molecules, so we pursue fractional STIRAP [1] via the Vitanov et al. 11] pulseforms: $\Omega_{+}(t)=\Omega_{0} \sin \alpha \exp \left[-(t-\tau)^{2} / T^{2}\right]$ and $\Omega_{-}(t)=$ $\Omega_{0} \exp \left[-(t+\tau)^{2} / T^{2}\right]+\Omega_{0} \cos \alpha \exp \left[-(t-\tau)^{2} / T^{2}\right]$, where $\tan \alpha$ sets the final population fraction. Using Fock states and the Hamiltonian given in the Appendix, we solve the Schrödinger equation numerically. The key results are presented in Fig. 1] For $N_{F}=4$ and $N_{B}=100$, the system reproduces the single particle case $\left(N_{B}=N_{F}=1\right)$, i.e., the results are identical to those for a three-level atom, as expected for a mostly-undepleted boson field. Atom-molecule collisions are negligible for couplings as large as $\Lambda_{+-} / \Omega_{0}=10^{-5}$, which is similar to a bosonic system, lending confidence to the restrictedcollision model. Furthermore, $N_{F}=N_{B}=4$ shows that many-body effects can limit molecular conversion. This many-body effect is similar to the one-color BEC

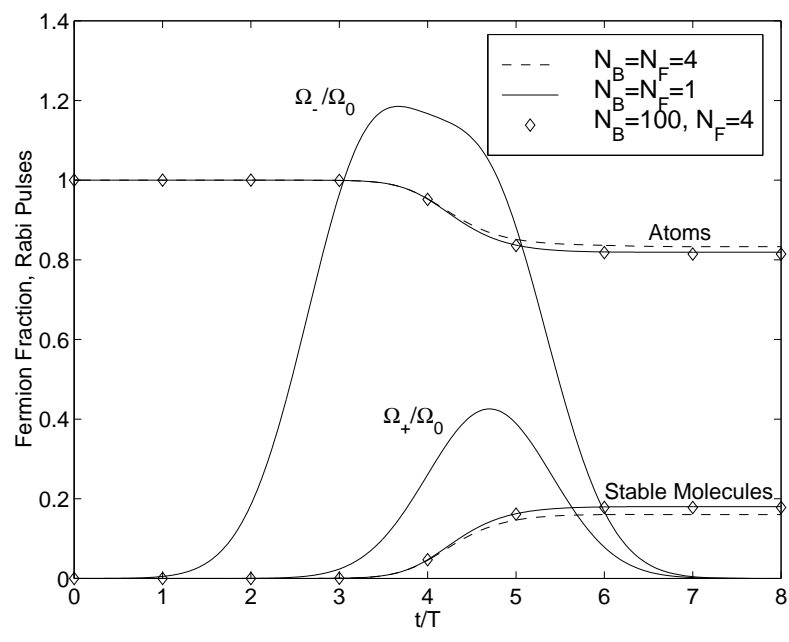

FIG. 1: Creation of an arbitrary mixture of Fermi degenerate atoms and molecules via fractional stimulated Raman adiabatic passage. Units of frequency are set by the choice $\Omega_{0}=2 \pi \mathrm{MHz}=1$, and the pulse parameters are $\alpha=0.14 \pi$, $T=5 \times 10^{3}$, and $\tau=0.7 T$. For $N_{B}=100$ and $N_{F}=4$, fractional STIRAP exhibits no visible difference from the $N_{B}=N_{F}=1$ case, while $N_{B}=N_{F}=4$ illustrates that many-body effects can limit the conversion efficiency.

case [15], and is not attributable to fermion statistics.

It is not entirely surprising to find a fermionic atoms responding as a unit to form fermionic molecules: cooperative fermionic behavior was predicted for one-color formation of Fermi molecules [16], as well as in atomic four-wave mixing [17] and, more relatedly, cooperation was observed in magnetoassociation of Fermi atoms into Bose molecules [18]. However, what is surprising is that STIRAP should work basically as expected even in the presence of collisions. This situation arises because, when $N_{B} \gg N_{F}$, the condensate density is effectively fixed, and the associated mean-field contribution simply amounts to a static bias that can be absorbed into the detuning; with Fermi-Fermi collisions blocked, which is most likely for small ground-state molecules, only collisions between the Fermi atoms and molecules can move the system off the required two-photon resonance, and STIRAP works better. In other words, we get the advantage of Bose enhancement of the free-bound coupling $\left(\Omega_{+} \propto \sqrt{\rho_{B}}\right)$, without the mean-field shifts. While limited computational resources preclude explicit investigation, these results ought to scale qualitatively with increasing particle number, as we have seen for up to $N_{F}=20$ in one-color production of Fermi molecules.

Now we are safe to presume the existence of an arbitrary admixture of Fermi-degenerate atoms and molecules, and thus to consider any subsequent anomalous pairing. Once the transient STIRAP pulses have vanished, the system is described by $\mathcal{H}_{0}$ [Eq. [1a)] with $\Delta=\delta=0$ and $\sigma=0, \pm$. For equal-mass fermions, it is known that a fermion density fluctuation gives rise to an effective chemical potential for the bosons, which cre- 
ates a bosonic density fluctuation, which in turn leads to an effective chemical potential for the fermions. In other words, phonons spawned by BEC density fluctuations are exchanged between the fermions, altering their interaction. Just like lattice vibrations that drive the attraction between degenerate electrons in superconductors, BEC density fluctuations lead to an attractive interaction that can enhance overall attractions, and thus Cooper pair formation [13].

Here the effective Fermi-Fermi scattering length is

$$
\bar{a}_{+-}=a_{+-}\left[1+\frac{\ln (4 e)^{2 / 3}}{\pi} k_{F} a_{+-}-H \frac{\lambda_{0+} \lambda_{0-}}{\lambda_{00} \lambda_{+-}}\right] \text {, }
$$

where $H=\ln \left(1+x^{2}\right) / x^{2}$ with $x=\hbar k_{F} / m_{0} v_{s}$ and $v_{s}=\left(\rho_{B} \hbar \lambda_{00} / m_{0}\right)^{1 / 2}$ is the speed of phonons in BEC; $\hbar k_{F} \ll m_{0} v_{s}$ implies $H \approx 1$. In other words, the effective scattering length can be written $\bar{a}_{+-} / a_{+-}=$ $1+\eta_{F F}-\eta_{F B}$, where $\eta_{F F}\left(\eta_{F B}\right)$ is the contribution to atom-molecule interactions from fermion-fermion (bosonfermion) fluctuations. Implicit to expression (2) is the perturbative assumption $\eta_{F B} \ll \eta_{F F}$. The immediate contrast with Ref. [13] is that $\eta_{F B}<0$ is allowed. For a weakly attractive system $\left(k_{F}\left|a_{\sigma_{1} \sigma_{2}}\right|, \rho_{B}\left|a_{\sigma_{1} \sigma_{2}}\right|^{3} \ll 1\right)$, the critical temperature for Cooper pairing is

$$
T_{c}=0.61 T_{F} \exp \left[-\frac{\pi / 4}{k_{F}\left|\bar{a}_{+-}\right|}\right],
$$

where $T_{F}=\hbar\left(\mu_{+}+\mu_{-}\right) R_{M} / k_{B}$ is the Fermi temperature with $R_{M}=m_{+-}^{*} / \sqrt{m_{+} m_{-}}$. The Fermi wavevector, $k_{F}$, was taken as the same for both species, so that $\mu_{+}+\mu_{-}=$ $\left(m_{ \pm} / m_{+-}^{*}\right) \mu_{ \pm}$and $T_{F}=T_{F}^{(+)} \sqrt{m_{+} / m_{-}}$.

Continuing to focus on ${ }^{87} \mathrm{Rb}^{40} \mathrm{~K}, N_{B}=1.3 \times 10^{6}$ BEC atoms in a trap with $\omega_{r}=100 \times 2 \pi \mathrm{Hz}$ and $\omega_{a}=10 \times 2 \pi \mathrm{Hz}$ yield a boson density $\rho_{B}=5 \times 10^{14} \mathrm{~cm}^{-3}$. A modest number of fermions, $N_{F}=5 \times 10^{3}$, means that the loss of condensate atoms in molecule formation can be neglected, the condensate will absorb any heat created by pairing-induced holes in the atomic Fermi sea [19], and collapse instabilities (Modugno et al. [10]) are avoided. Presuming that fractional STIRAP converts roughly $18 \%$ of the initial Fermi atoms into molecules (see Fig. 1), and that the fermions share the same (mass-adjusted) trap, then $\rho_{ \pm}=1.1 \times 10^{12} \mathrm{~cm}^{-3}$, and the requirement of equal Fermi wavenumbers for the atoms and molecules is met. For the given parameters, the size of the BEC is roughly an order of magnitude larger than the Fermi clouds, so that overlap should not be an issue.

Figure 2] summarizes our investigations. Under the above trap conditions, and for $\left|a_{+-}\right|=a_{00}=5.29 \mathrm{~nm}$, we find the weakness parameter $k_{F}\left|a_{+-}\right|=0.05$ and an unreasonably low critical temperature. Nevertheless, if the trap is modified to be anharmonic, then tighter confinement ultimately means a diluteness parameter on the order of $k_{F}\left|a_{+-}\right|=0.2$ [20], and the situation is improved but still out of reach of current technology $\left(T \sim 0.05 T_{F}[18]\right)$. The only chance appears to be for a tight trap and a large scattering length, $k_{F}\left|a_{+-}\right|=0.5$;

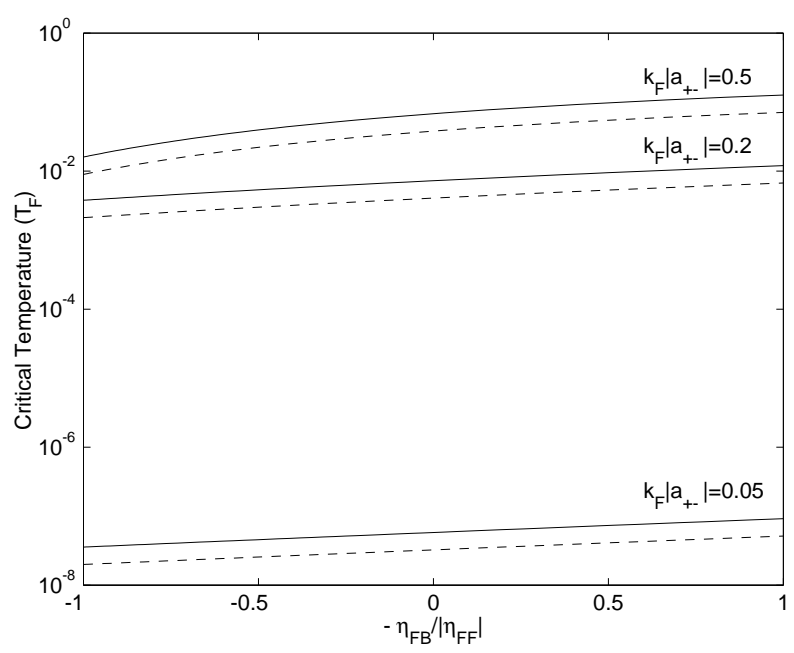

FIG. 2: Critical temperature for the superfluid transition to anomalous atom-molecule pairs as a function of fermion-boson fluctuation strength. Calculations are for weak $\left(k_{F}\left|a_{+-}\right|=\right.$ $0.05)$, marginally weak (0.2), and marginally strong (0.5) interactions. The solid (dashed) curve gives the critical temperature in units of the effective (atomic) Fermi temperature.

however, the theory is at best marginally applicable in this regime and, besides, some other process (e.g., threebody recombination) would probably win out before superfluidity could set-in to such a system.

In conclusion, collisions can arguably be made negligible in photoassociation of Bose-Fermi mixtures of atoms, and fractional stimulated Raman adiabatic passage scheme is therefore feasible for creating Fermi degenerate molecules. On the other hand, while possible in principle, accessible atom-molecule superfluidity means that next-generation technology must shed another order of magnitude in temperature from the first generation [21], or that a system with an attractive and a strong-but-not-too-strong interaction will be found. Our opinion is that it will be worthwhile to look for other ways of driving atom-molecule Cooper pairs, such as a photoassociation or Feshbach resonance.

Appendix.-To model STIRAP from Eq. (1), make a time-dependent unitary transformation $U(t)=\Pi_{\sigma} U_{\sigma}(t)$, where $U_{0(+)}(t)=\exp \left[-i \mu_{+(0)} \psi_{+(0)}^{\dagger} \psi_{+(0)} t\right], U_{e}(t)=$ $\exp \left[i\left(\mu_{+}+\mu_{0}\right) \psi_{e}^{\dagger} \psi_{e} t\right], U_{-}(t)=\exp \left[-i\left(\mu_{+}+\mu_{0}\right) \psi_{-}^{\dagger} \psi_{-} t\right.$, whereby $\mu_{e} \rightarrow \mu_{e}^{\prime}=\mu_{e}-\left(\mu_{-}+\mu_{0}\right)$ and $\mu_{-} \rightarrow \mu_{-}^{\prime}=$ $\mu_{-}-\left(\mu_{-}+\mu_{0}\right)$; in turn, absorb $\mu_{e(-)}^{\prime}$ into the intermediate (two-photon) detuning. Assume that atom-molecule conversion occurs on a timescale much faster than the motion of the atoms in the trap, and thereby neglect the kinetic energies and any explicit trapping potentials. Take the Fermi energy to lie within the Wigner threshold regime, so that the coupling $\mathcal{K}_{+}$is the same for all modes. Focus on the regime $N_{B} \gg N_{F}$, so that the condensate is practically undepleted and $\psi_{0} \approx \sqrt{\rho_{B}}$ can be absorbed into the atom-molecule coupling strength; likewise, the BEC mean-field shifts, $\lambda_{0 \sigma} \psi_{0}^{\dagger} \psi_{0} \approx \rho_{B} \lambda_{0 \sigma}$, are a constant 
that can be absorbed into the detuning, and therefore of no concern. Input two-photon and intermediate resonance by setting $\Delta=0$ and $\delta=-\frac{1}{2} i \Gamma$. Finally, switch to momentum space, so that

$$
\begin{aligned}
\frac{H}{\hbar}= & -\frac{1}{2} i \Gamma \sum_{\mathbf{k}} a_{\mathbf{k} e}^{\dagger} a_{\mathbf{k} e}+\Lambda_{+-} \sum_{\left\{\mathbf{k}_{i}\right\}} a_{\mathbf{k}_{1}+}^{\dagger} a_{\mathbf{k}_{2}-}^{\dagger} a_{\mathbf{k}_{3}-} a_{\mathbf{k}_{4}+} \\
& -\frac{1}{2} \sum_{\mathbf{k}}\left[\left(\Omega_{+} a_{\mathbf{k} e}^{\dagger} a_{\mathbf{k}+}+\Omega_{-} a_{\mathbf{k} e}^{\dagger} a_{\mathbf{k}-}\right)+\text { H.c. }\right],
\end{aligned}
$$

where $\Omega_{+}=\sqrt{\rho_{B}} \mathcal{K}_{+}$and $\Lambda_{+-}=\lambda_{+-} / V$. Recover the few-boson results by assuming that Bose stimulation favors condensate modes, and making the substitutions $\Omega_{+} \rightarrow \Omega_{+} / \sqrt{N_{B}}$ and $a_{\mathbf{k} e}^{\dagger} a_{\mathbf{k}+} \rightarrow a_{\mathbf{k} e}^{\dagger} a_{\mathbf{k}+} a_{0}$.

Acknowledgements.- The authors thank Emil Lundh, Chris Pethick, Mirta Rodriguez, Eddy Timmermans, and Päivi Törmä for helpful discussions; also, the Academy of Finland (MM and KAS; project 50314), the Magnus Ehrnrooth foundation (OD), NSF and NASA (JJ; PHY0097974 and NAG8-1428) for financial support.
[1] J. Weiner, V. S. Bagnato, S. Zilio, and P. S. Julienne, Rev. Mod. Phys. 71, 1 (1999).

[2] J. Bardeen, L. N. Cooper, and J. R. Schrieffer, Phys. Rev. 106, 162 (1957).

[3] M. Tinkham, Intro. to Superconductivity, (McGraw-Hill, New York, 1975).

[4] A. Bohr, B. R. Mottelson, and D. Pines, Phys. Rev. 110, 936 (1958).

[5] H. Heiselberg, physics/0304005

[6] A. Vardi, D. Abrashkevich, E. Frishman, and M. Shapiro, J. Chem. Phys. 107, 6166 (1997).

[7] M. Mackie, R. Kowalski, and J. Javanainen, Phys. Rev. Lett. 84, 3803 (2000).

[8] J. J. Hope, M. K. Olsen, and L. I. Plimak, Phys. Rev. A 63, 043603 (2001); P. D. Drummond, K. V. Kheruntsyan, D. J. Heinzen, and R. H. Wynar, Phys. Rev. A 65, 063619 (2002); M. Mackie, A. Collin, and J. Javanainen, physics/0212111

[9] B. Damski, L. Santos, E. Tiemann, M. Lewenstein, S. Kotochigova, P. Julienne, and P. Zoller, Phys. Rev. Lett. 90, 110401 (2003).

[10] A. G. Truscott, K. E. Strecker, W. I. McAlexander, G. B. Partridge, and R. G. Hulet, Science 291, 2570 (2001); F. Schreck, G. Ferrari, K. L. Corwin, J. Cubizolles, L. Khaykovich, M.-O. Mewes, and C. Salomon, Phys. Rev. A 64, 011402 (R) (2001); G. Modugno, G. Roati, F. Riboli, F. Ferlaino, R. J. Brecha, and M. Inguscio, Science 287, 2240 (2002).
[11] P. Marte, P. Zoller, and J. L. Hall, Phys. Rev. A 44, R4118 (1991); N. V. Vitanov, K.-A. Suominen, and B. W. Shore, J. Phys. B 32, 4535 (1999).

[12] K. Bergmann, H. Theuer, and B. W. Shore, Rev. Mod. Phys. 70, 1003 (1998).

[13] H. Heiselberg, C. J. Pethick, H. Smith, and L. Viverit, Phys. Rev. Lett. 85, 2418 (2000); M. J. Bijlsma, B. A. Heringa, and H. T. C. Stoof, Phys. Rev. A 61, 053601 (2000).

[14] U. Schlöder, C. Silber, T. Deuschle, and C. Zimmermann, Phys. Rev. A 66, 061403 (R) (2002).

[15] J. Javanainen and M. Mackie, Phys. Rev. A 59, R3186 (1999); A. Vardi, V. A. Yurovsky, and J. R. Anglin, Phys. Rev. A 64, 063611 (2001).

[16] O. Dannenberg, M. Mackie, and K.-A. Suominen, Phys. Rev. Lett. 91, 210404 (2003); M. Wouters, J. Tempere, and J. T. Devreese, Phys. Rev. A 67, 063609 (2003).

[17] M. G. Moore, and P. Meystre, Phys. Rev. Lett. 86, 4199 (2001); W. Ketterle, and S. Inouye, Phys. Rev. Lett. 86, 4203 (2001).

[18] C. A. Regal, C. Ticknor, J. L. Bohn, and D. S. Jin, Nature 424, 47 (2003); K. E. Strecker, G. B. Partridge, and R. G. Hulet, Phys. Rev. Lett. 91, 080406 (2003).

[19] E. Timmermans, Phys. Rev. Lett. 87, 240403 (2001).

[20] C. P. Search, H. Pu, W. Zhang, B. P. Anderson, and P. Meystre, Phys. Rev. A 65, 063616 (2002).

[21] B. DeMarco and D. S. Jin, Science 285, 1703 (1999). 